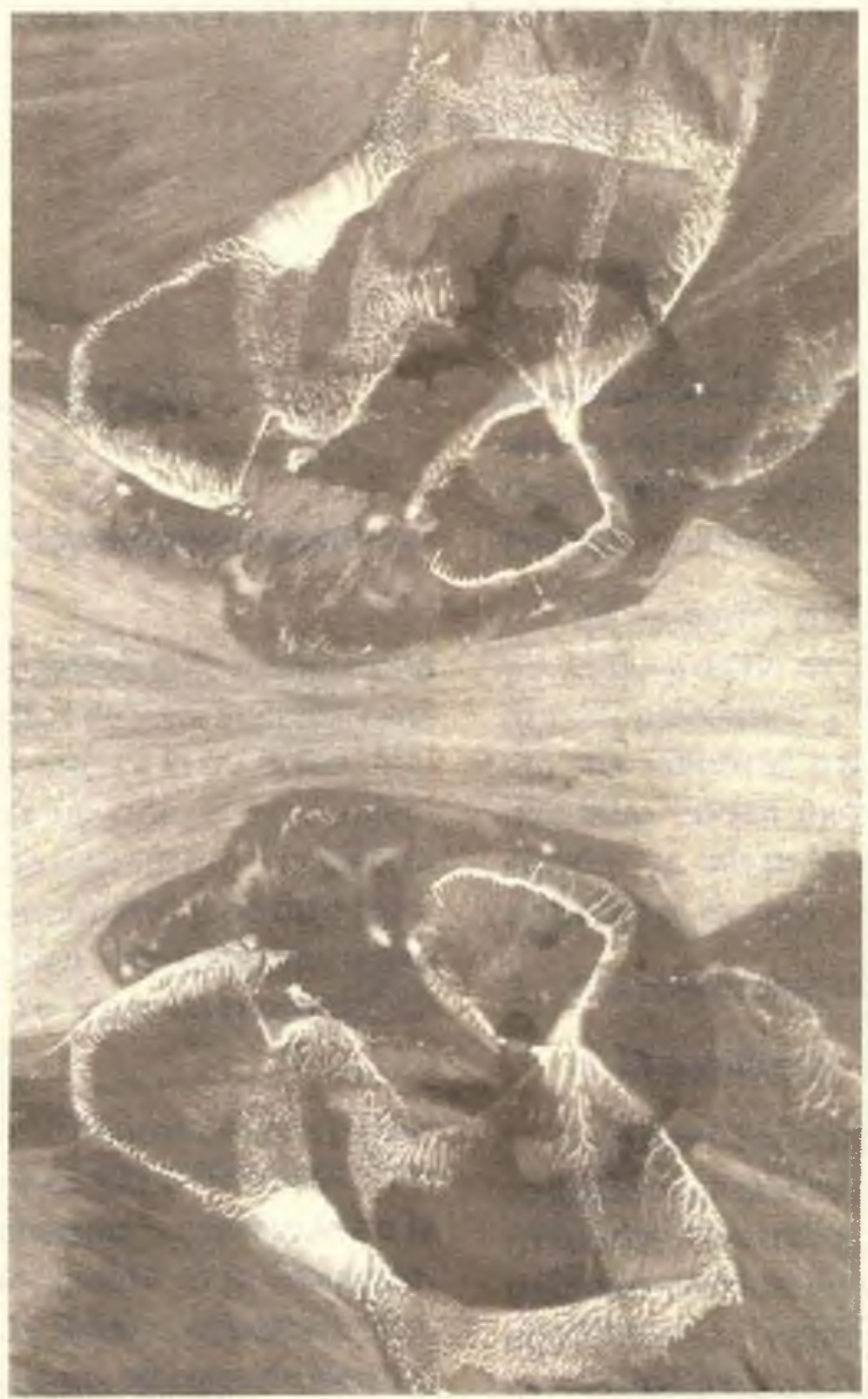

Ilustración: Carolina Ramírez V. 


\title{
EL ORDEN EMANADO DEL CAOS
}

\author{
ILYA PRIGOGINE *
}

\section{UNA CONTRADICCIÓN FUNDAMENTAL}

\section{Una contradicción del pensamiento oc- cidental}

De mi parte, he considerado siempre esta divergencia de puntos de vista manifestados como el corte más neto de la tradición intelectual europea. De un lado, el pensamiento europeo ha dado nacimiento a la ciencia y por tanto al determinismo y, de otro lado, él comprende tan bien al humanismo, quien lleva sobretodo a las ideas de responsabilidad y creatividad.

Esta diferencia de puntos de vista es hoy mas fuerte que nunca. Un artículo de la revista "Scientific American", consagrada a la vida en el universo, insiste en la evolución, en la Historia. Los físicos, opuestamente, evocan el determinismo, las leyes de Newton y la reversibilidad de éstas.

Por supuesto, yo no soy el primero en pensar que la ciencia no ha resuelto totalmente el problema del tiempo. Pero las actitudes del pasado eran diferentes de aquella que yo aprendí instintivamente. Pensadores como Bergson o Heidegger plantean que el tiempo es de la incumbencia de la metafísica y no de la física. Para ellos, el tiem-

- Nobel de química. Traducido del francés por Piedad Gooding po pertenece claramente a otro registro: la ciencia no tiene nada que decir del tiempo. Otros como Einstein, no ven en el tiempo mas que una ilusión.

De mi parte, yo considero que el tiempo se desprende de lo complejo. Una piedra del paleolítico y una piedra del XIX siglo son idénticas pero los edificios de las que ellas hacían parte no tienen nada en común: es mirando el todo que veremos aparecer el tiempo.

\section{Las tres etapas de la reflexión}

\section{- El equilibrio}

Yo quiero distinguir dos etapas en las investigaciones que conduzco. La primera corresponde a los trabajos que realicé hace una treintena de años, que mostraron que el noequilibrio era generador de tiempo, de irreversibilidad, de construcción.

Durante el siglo XIX y durante una gran parte del XX, los científicos estaban sobre todo interesados en los estados de equilibrio. Luego ellos han emprendido progresivamente el análisis de los estados próximos al equilibrio. Tenemos la tendencia a creer que la evolución hacia el equilibrio era sinónimo de pérdida de la información, de una uniformización del sistema. Pero, desde que uno se aleja así sea un poco del equilibrio, asistimos a la coexistencia de fenómenos de orden y desorden. Una ilustración de esta 
coexistencia es la termodifusión. No podemos pues asimilar la irreversibilidad al desorden.

\section{- El no-equilibrio}

Tal era pues la primera etapa de mi reflexión: el equilibrio. Abordemos ahora la segunda etapa, el no-equilibrio lineal. Alejarse del equilibrio reserva sorpresas. En efecto, es imposible mantener aquello que sabemos sobre el equilibrio. Descubrimos así nuevas situaciones, a veces mas organizadas que en el equilibrio.

Aquello se produce en puntos particulares, que corresponden a los cambios de fase del no-equilibrio, que yo llamo puntos de bifurcación. Una ecuación no lineal admite generalmente varias soluciones: el entorno del equilibrio es una de ellas. Es la solución que dará, al equilibrio, el mínimo de energía libre, el máximo de entropía y así sucesivamente. Contrariamente, lejos del equilibrio, esta solución puede llegar a ser inestable. Este género de bifurcaciones se produce, en química, cuando las ecuaciones son no lineales y que contienen elementos autocatalíticos, es decir feed-back positivos. Luego de la aparición de esos rompimientos, constatamos que las nuevas soluciones aparecen. Y, por supuesto, cuando cambiamos el parámetro de control, podemos obtener aún otras soluciones: no hay una sola bifurcación sino un conjunto de bifurcaciones.

Así, el no-equilibrio es creador de estructuras, llamadas disipativas porque no existen sino lejos del equilibrio y porque ellas exigen la disipación de energía, por lo tanto un mantenimiento de interacción con el mundo exterior, para sobrevivir. Como una ciudad que no existe en tanto que funcione y que mantenga intercambios con el exterior, la estructura disipativa desaparece cuando no es "alimentada".

Ha sido pues muy sorprendente descubrir que, lejos del equilibrio, la materia tie- ne propiedades nuevas; igualmente, la variedad de los comportamientos posibles es muy sorprendente. Las reacciones químicas oscilantes han sido una de las primeras aplicaciones. A propósito, no puedo dejar de pensar que la publicación de nuestros trabajos se hace en un momento particularmente feliz, pues al mismo tiempo, las experiencias sobre las moléculas coloreadas de Japoutinsky han sido publicadas. Tendemos, intuitivamente, a pensar que una reacción química es un fenómeno desorganizado, donde el azar juega un gran rol. Pero estas experiencias han probado la existencia de fenómenos organizados.

El no-equilibrio desemboca pues en una nueva coherencia, en fenómenos ondulatorios, donde el milagro es que poseen longitudes de onda característicos. La mecánica cuántica es célebre por sus fenómenos ondulatorios asociados al electrón; tenemos aquí fenómenos ondulatorios ligados a la química. Hay otros casos en los que las moléculas no están repartidas uniformemente sino formando islotes, aquello que llamamos una cristalografía de no-equilibrio. Estos fenómenos no son estudiados únicamente en química sino también en óptica, en hidrodinámica, los vemos en neurofisiología.

En equilibrio, la materia es ciega, mientras que lejos del equilibrio, ella busca correlaciones de largo alcance. Finalmente, las situaciones cercanas del equilibrio son estables y caracterizadas por un mínimo de cualquier cosa ( energía, entropía, etc.). En ese caso, si usted perturba aunque sea un poco el sistema, este regresa automáticamente al equilibrio: las eventuales fluctuaciones mueren por sí mismas.

Contrariamente, lejos del equilibrio, ese mínimo de energía libre no existe mas, de tal suerte que las fluctuaciones pueden amplificarse. Podemos así considerar las estructuras disipativas como fluctuaciones gigantes estabilizadas. Es pues un mundo 
mucho mas complejo y variado el que se observa lejos del equilibrio.

Todos estos fenómenos existen en biología: los biólogos, los neurofisiólogos estudian de cerca los fenómenos no lineales de estructuración. Así, las señales temporales del cerebro deben ser caóticas, en defecto de lo cual se observan síntomas de enfermedades tales como la epilepsia.

- El no-equilibrio, fuente de estructura

Llegamos, en total, a esa conclusión, paradojal que quiere que el no equilibrio sea fuente de estructura. Esta evolución apenas ha comenzado. En efecto, el no-equilibrio es una interfase entre la ciencia pura y la aplicada, si bien las aplicaciones de esas observaciones a la tecnología están sólo en el comienzo. En el fondo, uno se da cuenta de que la naturaleza utiliza el no-equilibrio para sus estructuras más complejas. El pasaje de la materia a la vida ha sido objeto de debates apasionados. Comenzamos actualmente a comprender que la vida es probablemente la confluencia de una evolución que se dirige hacia sistemas cada ves más complejos. Es verdad que uno no sabe exactamente qué mecanismo ha producido las primeras moléculas capaces de reproducirse por sí mismas. La vida tiene una tecnología admirable, que se nos escapa frecuentemente y en relación a la cual la nuestra es muy primitiva.

El no-equilibrio no puede ser formalizado por el sesgo de ecuaciones deterministas. De hecho, las bifurcaciones son numerosas $\mathrm{y}$, cuando se repiten las experiencias, el "camino" seguido no es siempre el mismo. Así, el fenómeno es determinista entre las bifurcaciones pero es totalmente aleatorio en el punto de las bifurcaciones.

Dos aspectos fundamentales aparecen netamente en los fenómenos de no-equilibrio. Se trata, de una parte del aspecto creativo y constructor del tiempo y, de otra parte, del aspecto aleatorio. Este último aparece particularmente claro en el caos determinista, en el cual las soluciones a ecuaciones muy simples, deterministas, son inestables.

Estos dos aspectos, se conciben claramente, se sitúan en contradicción directa con las leyes fundamentales de la física enunciadas por Newton y por Einstein quienes niegan el indeterminismo y la dirección del tiempo. Esas leyes fundamentales quedan por tanto no narrativas, estáticas. Esta con-

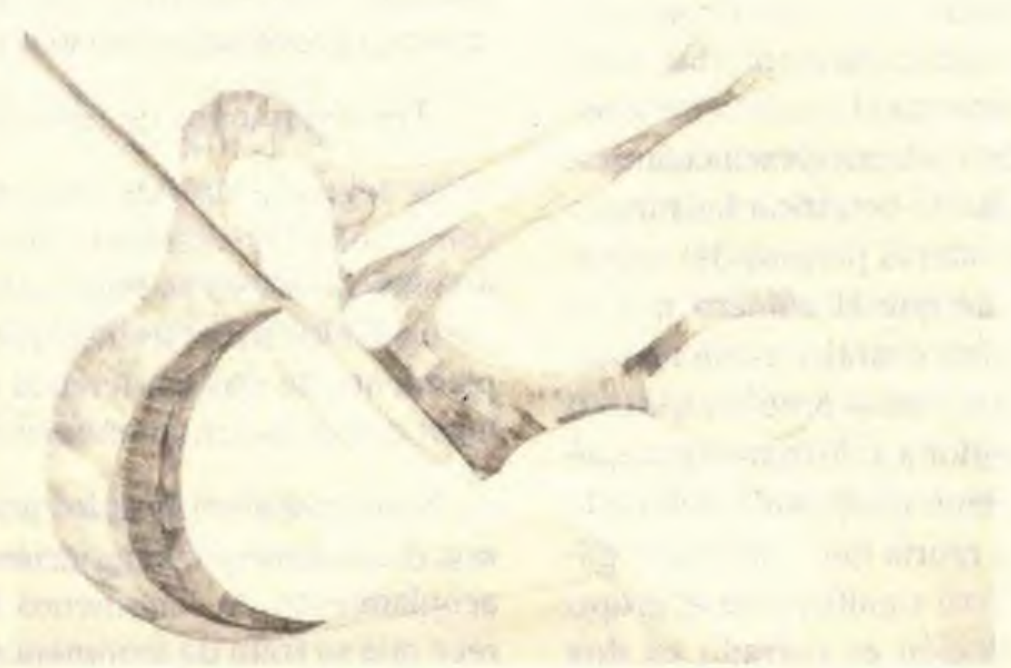

llustración: Guillermo Ramírez 
tradicción me ha evidentemente preocupado bastante.

\section{II. ¿CÓMO SOBREPASAR ESTA CONTRADICCIÓN?}

La teoría dinámica ha hecho grandes progresos y nos ofrece de esta manera instrumentos particularmente interesantes. Se estima que Newton resolvió el problema de la dinámica poniendo las ecuaciones donde no quedaba más que encontrar las soluciones. Pero contrariamente a lo que pensaba Newton, sabemos ahora que todos los sistemas dinámicos no son idénticos. Distinguimos dos tipos de sistemas: los sistemas estables y los sistemas inestables.

\section{El caos determinista}

Separamos además los sistemas inestables en dos categorías diferentes. Los sistemas más simples son ordenados bajo el nombre de caos determinista. En el caos determinista, encontramos situaciones en donde las ecuaciones de base son deterministas pero las soluciones toman un aspecto aleatorio. Esto se explica por la sensibilidad a las condiciones iniciales: la mínima modificación de las condiciones iniciales prepara divergencias exponenciales.

¿Cómo tratar el mundo inestable? Conviene en su lugar pensar en términos de trayectoria, en términos de probabilidades. Se hace entonces posible hacer predicciones por grupos de sistemas. La teoría del caos es en esto parecida a la mecánica cuántica. Hay que, allí también, identificar las funciones propias y los valores propios del operador. Pero, luego de que el número que se obtiene en mecánica cuántica tiene un módulo 1, el módulo del valor propio en la teoría del caos es inferior a 1. Esto muestra que el futuro es diferente del pasado y sitúa la necesidad de una teoría que distinga el pasado del futuro. Esto significa que el grupo dinámico de evolución es cortado en dos semi-grupos, en donde uno se acerca al equilibrio y el otro se aleja. Nosotros no debemos retener sino el primero, el cual corresponde al hecho de que el futuro es común a todos: la unidad del tiempo es el elemento común del universo.

La flecha del tiempo, así, es a la vez el elemento común del universo y el factor de distinción entre lo estable y lo inestable, entre lo organizado y el caos. Para ir mas lejos en la reflexión sobre el caos determinista, es necesario sobrepasar la física cuántica, y notablemente salir del espacio euclidiano en el seno del cual ella ha sido definida. Felizmente, matemáticos franceses luego rusos, a la cabeza de los cuales Laurent Schwartz, han descrito una nueva matemática, que permite introducir los fenómenos del caos determinista. Esta nueva matemática ha pues servido a la elaboración de nuestra teoría.

Pero el caos determinista no explica todo. La historia, la economía no se prestan siempre a la aplicación de la teoría, porque ellas son inestables: presentan la apariencia de caos pero no están fundamentadas sobre ecuaciones de base deterministas. El proceso de la toma de decisiones, esencial en la vida de una empresa, llama a factores complejos (historia pasada, anticipación al futuro) que engendran una dislocación en el tiempo; las operaciones bursátiles proporcionan un ejemplo muy pertinente de situaciones donde ensayamos proyectarnos en el futuro.

\section{Los fenómenos de resonancia}

El segundo tipo de sistemas inestables comprende los sistemas conocidos bajo el nombre de sistemas Poincaré. Ellos son en particular los sistemas de resonancia: el acoplamiento de dos fenómenos dinámicos da lugar a nuevos fenómenos dinámicos.

Yo ensayé identificar los nuevos fenómenos dinámicos que se podrían obtener por acoplamiento de fenómenos Poincaré. Parece que se trata de fenómenos de difusión. Los fenómenos de resonancia introducen la 
indeterminación en la fortaleza determinista de la mecánica clásica y tan bien en la fortaleza de la mecánica cuántica. Nosotros nos hemos esforzado, en el transcurso del último año, en observar esos nuevos fenómenos. Se trata allí de un ejercicio difícil, en la medida en que ellos muestran distancias en relación a las leyes de Newton.

No hay que esperar, evidentemente, encontrarlos por todas partes: los principios de la mecánica clásica y de la mecánica cuántica han sido verificados en numerosos dominios. Hace falta, pues, identificar las situaciones nuevas. Un primer ejemplo nos es presentado por la colisión persistente. Hemos igualmente estudiado aquello que serían los sistemas de Poincaré en el límite termodinámico, es decir luego que el número de partículas tiende hacia el infinito y que la relación volumen-partículas sigue constante. Las partículas, en efecto, están en interacción continua, lo que conduce a nuevos fenómenos, tales como líquidos o gases que no existen sino en el límite termodinámico. Las correlaciones de Poincaré crean vínculos entre esas partículas $y$, al infinito, divergencias, fenómenos aleatorios pueden introducirse. Es en ese momento que podemos observar las distancias en relación a Newton, bajo la fonna de fenómenos de difusión o de fenómenos irreversibles. Esta aparición de fenómenos irreversibles muestra que uno puede tirar un puente entre la termodinámica, los fenómenos macroeconómicos y los fenómenos elementales. Esto presenta un gran interés práctico. Así, los fenómenos de disipación son tratados de manera empírica; pero hoy, comenzamos a poder formalizar, aquello que tendrá aplicaciones técnicas interesantes.

\section{CONCLUSIÓN}

Nos encontramos en un periodo interesante de la ciencia, que corresponde a la apa- rición de una nueva visión del mundo. Hasta ahora, el pensamiento ponía el acento sobre la estabilidad y el equilibrio. Esto no es nada. Newton mismo presentía la inestabilidad del sistema planetario pero encontró esta idea tan absolutamente insoportable que la alejó, fiándose de la intervención del Agente, Dios, quien repararía su propia creación.

Hoy, somos capaces de abstraernos de los prejuicios de Newton (al menos de algunos) y debemos integrar la idea de inestabilidad del universo. La inestabilidad no debe conducirnos al inmovilismo. Al contrario, debemos estudiar las razones de esta inestabilidad, con el fin de describir el mundo en su complejidad y de reflexionar sobre la manera de actuar sobre la inestabilidad. Tal es la misión de los ingenieros y de los físicos. Karl Popper decía que existe la física de los relojes y aquella de las nubes. Después de haber estudiado la física de los relojes, nosotros debemos ahora estudiar la física de las nubes...

La física clásica insistía sobre la permanencia y el equilibrio. Sin embargo, ella daba una visión dualísta del mundo, en la cual el Hombre, un autómata, era separado del mundo. La nueva física acepta la complejidad del mundo y considera que el Hombre amplifica los fenómenos presentes en el mundo: la inestabilidad, la creatividad, la novedad. Eliminamos así la contradicción fundamental del dualismo cartesiano. Esta nueva visión del mundo me parece mejor acordada a nuestro fin de siglo, a la vez complejo y unificante. El equilibrio y la permanencia ocultaban las disparidades y el inmovilismo. Nuestra visión, al contrario, aproxima el Hombre a la naturaleza y es optimista, en este sentido es que esperamos aún importantes progresos en la comprensión del universo. 